\title{
Contention Aware Mobility Prediction Routing for Intermittently Connected Mobile Networks
}

\author{
Ahmed Elwhishi*, Pin-Han Ho*, and Basem Shihada ${ }^{\dagger}$ \\ * Dept. of Electrical and Computer Engineering, University of Waterloo, Canada, \{aelwhish, p4ho\}@uwaterloo.ca \\ $\dagger$ Computer Electrical Math Sciences \& Engineering, KAUST, Thuwal, Saudi Arabia, basem.shihada@kaust.edu.sa
}

\begin{abstract}
This paper introduces a novel multi-copy routing protocol, called Predict and Forward (PF), for Delay Tolerant Networks (DTNs), which aims to explore the possibility of taking mobile nodes as message carriers for end-to-end delivery of the messages. With PF, the message forwarding decision is made by manipulating the probability distribution of future intercontact and contact durations based on the network status, including wireless link condition and nodal buffer availability. In particular, $\mathrm{PF}$ is based on the observations that the node mobility behavior is semi-deterministic and could be predicted once there is sufficient mobility history information. We implemented the proposed protocol and compared it with a number of existing encounter-based routing approaches in terms of delivery delay, delivery ratio, and the number of transmissions required for message delivery. The simulation results show that the proposed PF outperforms all the counterpart multi-copy encounter-based routing protocols considered in the study.
\end{abstract}

Index Terms-Encounter-based Routing, DTN.

\section{INTRODUCTION}

DTNs [1] are characterized by the lack of end-to-end paths for a given node pair for extended periods, which demonstrates a complete different design scenario from that for the conventional mobile ad-hoc networks (MANETs) [2]. Due to the intermittent connections in DTNs, a node is allowed to buffer a message and wait until it finds an available link to the next hop that will be able to store the message. This process is repeated until the message reaches its destination. This model of routing constitutes a significant departure from that employed in MANETs. This new aspect of routing is usually referred to as encounter-based, store-carry-forward, or mobility-assisted routing, due to the fact that nodal mobility serves as a significant factor for the forwarding decision of each message.

Depending on the nodal mobility, the routing strategies in DTNs can be classified into three categories: precise (scheduled) contact routing, opportunistic contact routing, and predicted contact routing.

In scheduled routing strategies, the future network's state/topology is predictable, hence allowing message forwarding to be scheduled ahead of time. The space-time [3], modified shortest path [4], and interplanetary internet [5] are examples of scheduled routing schemes.

In opportunistic routing, there is no information available about contact time or place, and the connection is simply established at every contact opportunity, such as floodingbased schemes [6], [7] that distribute unlimited numbers of copies throughout the network, or controlled tree-based flooding schemes [8] that distribute only a subset of message copies are considered the main techniques for opportunistic routing. This type of routing is considered simple and suitable for the random nodal mobility scenarios, at the expense of extensively consumed resources as well as the possible performance degradation when the nodal mobility is not random.

In predicted routing, the prediction of a contact of each node pair is predicted based on some statistical information such as the last time of meeting, the frequency of meetings among nodes, the number of previous contact, or the probability of meeting between nodes in some locations. The techniques that employ this kind of mobility exploitation are also referred as utility-based routing [9], [10], [11], [12], [13], [14]. The decision of message forwarding to the encountered node is simply based on a developed utility function.

Although utility based routing schemes have improved in terms of performance, the previously reported schemes are subject to respective problems and implementation difficulties. Most importantly, these schemes inevitably take a large amount of transmission bandwidth and nodal memory space, which could easily dominate the network resource consumption [15]. Further, they suffer from contention in case of high traffic loads, in which packet drops could result in a significant degradation of performance and scalability. Note that the future DTNs may operate on a vast number of miniature and hand-held devices such as smart phones, tablet computers, personal digital assistants (PDAs), and fixed/mobile sensors, which are subject to a stringent limitation on power consumption and computation resources.

The paper introduces a novel DTN routing protocol, called Predict and Forward (PF), aiming to overcomes the shortcomings of the previously reported utility schemes. The main features of the proposed protocol are: (i) employing an efficient prediction of contact based on the probability distribution of meeting between nodes at different network partitions, (ii) capability to adapt to fluctuating network status, traffic patterns/characteristics, and user behaviors, so as to reduce the number of transmissions, message delivery time, and increase delivery ratio. This is achieved by jointly considering node mobility statistics, congestion, and buffer occupancy, which are subsequently fused in a novel quality-metric function. In specific, the link availability and buffer occupancy statistics are obtained by sampling the channels and buffer space during each contact with another node. The developed quality- 
metric function facilitates decision making for each active data message, resulting in optimized network performance. We will show via extensive simulations that the proposed PF can achieve a significant performance gain over the previously reported counterparts.

The rest of the paper is organized as follows. Section II gives a review over the related work. Section III describes the system model. Section IV introduces the method of estimating the contact probabilities. Section V introduces the proposed PF in detail. Then, Section VI provides the simulation results and the comparisons with the other counterparts. In Section VII, we conclude the paper.

\section{RELATED WORK}

The previously reported encounter-based routing protocols have focused on the node mobility which is exploited and taken as the dominant factor in the message forwarding decision. Those schemes contribute to the research community by introducing novel interpretations of the observed node mobility in the per-node utility function. Spyropoulos et al. in [14], [15] developed routing strategies using different utility routing metrics based on nodal mobility statistics, namely Most Mobile First (MMF), Most Social First (MSF) and Last Seen First (LSF). S. Nelson et al. [16] proposed an enhanced version of MSF, where the number of message replicas that are transferred during a contact is proportional to per-node utility function based on the evolution of the number of encounters a node has during a time-window. Lindgren et al. in [9] introduced a routing technique in DTNs which takes advantage of the predicted encounter probability between nodes. Jones et al. in [17] introduced a utility function for DTN routing which manipulates the minimum expected inter-encounter duration between nodes. Ling et al. in [18] designed a feedback adaptive routing scheme based on the factors solely determined by the node mobility, where a node with higher mobility is given a higher factor, and messages are transmitted through nodes with higher influence factors. A. Balasubramanian et al. in [10] considered statistics of available bandwidth and the number of message replicas currently in the network in the derivation of the routing metric to decide which message to replicate first among all buffered messages in a node.

Another scheme is called delegation forwarding [13], [19], where a custodian node forwards a message copy to an encountered node that has a better chance to "see" the destination. The key idea is that a custodian node (source or relay) forwards a message copy only if the utility function (represented by the rate of encounters between node pairs) of the encountered node is higher than all the nodes so far "seen" by a message, and then current custodian will update its utility value of that message to be equal to that of the encountered node.

The idea of routing using a node mobility profile is introduced by S. Merugu et al. in [3]. It is assumed that each node has a set of usually visited locations, referred to as the hub list of the node, which should be subject to very limited variation. The introduced protocol routes a message to one or more locations that have been visited by the destination when either the current message custodian or the encountered node visits these hubs. The contact probability between two nodes is computed based on all nodes's hub-visited probabilities.

It is assumed that each node knows its next hub, so that a node forwards a message to a number of its neighbors with a higher probability to visit a hub visited by the destination. Each node is assumed to know every other node mobility, and can compute the contact probabilities with every other node. Such contact relation is further abstracted into a weighted graph, wherein k-shortest path algorithm is applied to find a list of paths to every other destination. Although interesting, this mechanism of forwarding is subject to high overhead; and the assumption of knowing the complete topology regarding the nodal mobility is not realistic in most cases.

In the same line of [3], Q. Yuan et al. in [12] introduced the idea of the contact probability as the main factor of message forwarding. They introduced a single-copy routing scheme called predict and relay (PER) in the scenario where the node movement, including the information on when the contact will occur and how long it will last, is semi-deterministic and can be predicted provided with sufficiently long mobility history. By using a time-homogeneous semi-Markov process model, PER enables each node to keep the complete topology about every other node in the network. Obviously, such a scheme introduces extremely high computation complexity and operational overhead.

To countermeasure the deficiency of the previously reported designs, a number of studies were reported [15], [13], [20], [21], [22]. Nonetheless, they are subject to various limitations due to the simplified assumptions related to the nodal mobility scenarios [12], [23], or the utility function presentation [11]. More importantly, the channel capacity and buffer occupancy states have not completely been considered as factors in the derivation of utility functions. These two factors could be overlooked/ignored if the encounter frequency is low, where the network resource availability does not plays an important role and the routing protocol performance is dominated by node mobility. However, in the scenario that the nodal encounter frequency is large and each node has many choices for packet forwarding, the network resource availability could become a critical factor for improving routing protocol performance, and should be taken seriously in the derivation of utility functions.

\section{System Model}

As addressed in literature, many collected real world traces demonstrated that the nodes follow a semi-deterministic trajectory, or have some patterns in their movements [14], [10], [24].

To characterize the mobility pattern, in this work, we consider a partitioned community-based DTN with super node architecture [23]. In this model, a finite number of mobile nodes roam freely between a set of communities, each representing a MANET. A community is defined as a group of nodes who can communicate directly with each other, i.e., any two nodes located in a community within the radiation range 
of each other can establish a contact to exchange messages. Communities are also assigned unique IDs. Each community is attached to a supper node, which keeps all contact probabilities of the nodes that belong to the community. It also keeps track of the contact probability of any roaming node visiting its community. We assume each node is aware of which community it is located at anytime.

In this mobility model, the geographical service area is partitioned to $m$ communities. We consider only in-community meetings among nodes, on-way meetings are negligible due to relatively short meeting time. By assuming the node future location is independent of its past location given its current location, and each node has an exponential residence time for staying in a community or roaming between communities, the nodal mobility can be modeled as a continuous time Markov chain.

Specifically, the residence time of node $A$ in a community $C_{i}$ in each visit is an exponential random variable with parameter $\lambda_{i_{A}}^{l}$. When Node $A$ leaves Community $C_{i}$, it may move to any other community $C_{j}$, with a probability of $P_{C_{i j}}^{A}$. The roaming time of node $A$ from a community $C_{i}$ to any other community $C_{j}$ is exponentially distributed with parameter $\lambda_{i, j_{A}}^{r}$. Denote the location state of a mobile node $A$ by its current community, then the user mobility model can be characterized by a one-dimensional continuous-time Markov chain, with a location state space given by $C_{1}, C_{2}, \ldots, C_{m}$. The user movement model over the network coverage area is described by the transition matrix $M$ of the Markov chain, given by $P_{C_{i j}}^{A}$, which is the conditional probability that a mobile node enters community $C_{j}$ given that it is leaving its current community $C_{i}$. For any community $C_{i}$, we have $\sum_{j} P_{C_{i j}}^{A}=1$. The transition probability matrix depends on the geographical characteristics of the service area and the network environment under consideration.

\section{Estimation of Contact Probabilities}

Define inter-meeting time between a pair of nodes as the duration of time instant at which two nodes move out of each other's transmission range until the instant that they move within each other's transmission range the next time. Define meeting time as the duration of time in which two nodes remain in contact until they move out of transmission range of each other. Define node inter-arrival time for a community as the duration from the instant that a node departs from a community to the instant that the node arrives at the same community next time.

Our objective is to analyze the abovementioned node mobility model so as to obtain an accurate estimate of the contact probability between each node pair, where the nodal intermeeting and meeting times are characterized and focused. In following we derive the distribution of the node inter-arrival time for a community and the distribution of the inter-meeting time.

Inter-arrival Time (theory): Define $N(t)$ as the number of all visited communities by time $t$ for node $A$. Then, $N(t)$ is a non-homogeneous Poisson process with mean $=\int^{t} \lambda_{i}(s) d s$. This mean is the sum of the expected residence and roaming times for node A while it roams from one community to another. Define $N_{i}(t)$ as the number of visits by node $A$ to community $C_{i}$ by time $t$. Then, the mean arrival time to the $n^{\text {th }}$ community is $E_{A}^{n}=\sum_{i=1}^{n}\left(E_{i}[T c]+E_{i j}[T r]\right)$, where $E_{i}[T c]$ and $E_{i j}[T r]$ represent the average residence time at community $C_{i}$, and the average roaming time when node $A$ move from community $C_{i}$ to community $C_{j}$, respectively. In specific, node $A$ stays at $C_{i}$ for an exponential time with parameter $\lambda_{i_{A}}^{l}=\frac{1}{E_{i}|T c|}$ and roam with probability $P^{A} c_{i, j}$ to another $C_{j}$ for an exponential time with parameter $\lambda_{i, j_{A}}^{r}=\frac{1}{E_{i}[T r]}$, then the rate that node $A$ makes a transfer from state $i$ to state $j$ is calculated as

$$
\lambda_{i, j_{A}}=\frac{1}{1 /\left(\lambda_{i_{A}}^{l} P c_{i, j_{A}}\right)+\left(1 / \lambda_{i, j_{A}}^{r}\right)}
$$

The expected inter-arrival rate of node $A$ at $C_{i}$ is calculated as

$$
\lambda_{i_{A}}=1 /\left(\sum_{k=1}^{n} \frac{1}{\lambda_{i, j_{A}}^{k}}\right)
$$

where $n$ is the total number of visited communities by node $A$ until it come back to $C_{i}$.

Since the continuous-time Markov chain of node $A$ is irreducible, the limiting probabilities exist and can be obtained by solving $\pi_{A} Q_{A}=0$, and $\sum_{i} \pi_{A_{i}}=1$,

where $Q$ represents the generator matrix, which is given by

$$
Q_{i, j_{A}}=\left\{\begin{array}{cc}
\lambda_{i, j_{A}} & \text { if } i \neq j \\
-\sum_{j} \lambda_{i, j_{A}} & \text { if } i=j \\
0 & \text { otherwise }
\end{array}\right.
$$

$\pi_{A_{i}}$ is the limiting probability representing the fraction of time in which node $A$ resides in community $C_{i}$.

Thus, the parameter of inhomogeneous Poisson process, $N(t)$, can be presented as $\lambda_{i_{A}} \pi_{A_{i}} t$. As a result, the interarrival time of node $A$ to community $C_{i}$ is exponential with parameter $\lambda_{i_{A}} \pi_{A_{i}}$, and its mean is

$$
E_{i_{A}}=1 /\left(\lambda_{i_{A}} \pi_{A_{i}}\right)
$$

Inter-meeting Time (thoery): Nodes $A$ and $B$ meeting at $C_{i}$ can occur in two scenarios: (i) node $A$ moves to $C_{i}$ while node $B$ already resides in community $i$; (ii) node $B$ moves to $C_{i}$ while node $A$ already resides in $C_{i}$. Considering scenario (i), the number of meetings between the two nodes at $C_{i}$ is the fraction of node $A$ arrivals to community $C_{i}$ while node $B$ is residing there. According to the thoery of the inter-arrival time and the fact that node $B$ resides in community $C_{i}$ with probability $\pi_{B_{i}}$, the number of effective meetings between $A$ and $B$ at $C_{i}$ when node $A$ makes the movement follows Poisson process with mean $\lambda_{i_{A}} \pi_{A_{i}} \pi_{B_{i}} t$. Hence, the inter-meeting time between $A$ and $B$ at community $C_{i}$ when node $A$ makes the movement is an exponential 
random variable with parameter $\lambda_{i_{A}} \pi_{A_{i}} \pi_{B_{i}}$. Similarly, in scenario (ii), the inter-meeting time between $A$ and $B$ at $C_{i}$ when node $B$ makes the movement is an exponential random variable with parameter $\lambda_{i_{B}} \pi_{B_{i}} \pi_{A_{i}}$. As a result, the intermeeting time between $A$ and $B$ at $C_{i}$ is a random variable that is the minimum of the two independent exponential random variables, which follows an exponential distribution with parameter $\left(\lambda_{i_{A}} \pi_{A_{i}} \pi_{B_{i}}+\lambda_{i_{B}} \pi_{B_{i}} \pi_{A_{i}}\right)$. Considering all network partitions, the inter-meeting time between $A$ and $B$ is a random variable with a distribution of the minimum of the two nodes inter-meeting times at all the network partitions, which is an exponential random variable with parameter given by

$$
\sum_{i=1}^{m}\left(\lambda_{i_{A}}+\lambda_{i_{B}}\right) \pi_{A_{i}} \pi_{B_{i}}
$$

Noticeably, the above relation is largely based on the amount of meeting time intervals between nodes, which is in turn affected by the status and the duration of the status of the wireless channel and buffer. These contact components are time varying and could change dramatically from time to time. The short period of contact time or the unavailability of the channel or the buffer space may cause unsuccessful message exchange.

Thus, two factors should be considered in (5): (i) the impact the contact time duration. This factor is introduced as the probability that node $A$ will leave the neighborhood of community $C_{i}$ on or before time unit $k$, denoted as $S_{i}^{A}(k)$. Note that $S_{i}^{A}(k)$ also indicates the distribution of the sojourn time at $C_{i}$ for node $A$ regardless of the next community. The time $\operatorname{parameter}(k)$ is used as a relative time offset. (ii) The probability in which the channel is free and the buffer at the encountered node $B$ is not full at $C_{i}$, denoted as, $P_{f_{i}}$. This probability represents the probability of performing a successful message exchange when node $A$ encounters node $B$. By injecting the two parameters, (5) is reformulated as

$$
\sum_{i=1}^{m}\left(\lambda_{i_{A}}+\lambda_{i_{B}}\right) \pi_{A_{i}} \pi_{B_{i}} S_{i}^{A} P_{f_{i}}
$$

Consider two nodes, $A$ and $B$. Let $P_{A B}$ denote the probability that a contact occurs between the two nodes, given the expected residence time of both nodes in the network $R_{m}$. The probability of a contact based on the inter-meeting time between the nodes is calculated as

$$
P_{A B}=1-e^{-\sum_{i=1}^{m}\left(\lambda_{i_{A}}+\lambda_{i_{B}}\right) \pi_{A_{i}} \pi_{B_{i}} S_{i}^{A} P_{f_{i}} R_{m}}
$$

The contact probability between a super node $H$, node $A$ is calculated as

$$
P_{A H}=1-e^{-\lambda_{i} \pi_{A_{i}} S_{i}^{A} P_{f_{i}} R_{m}}
$$

The aforementioned relations represent the utility function maintained at each node regarding every other node and every super node in the network.

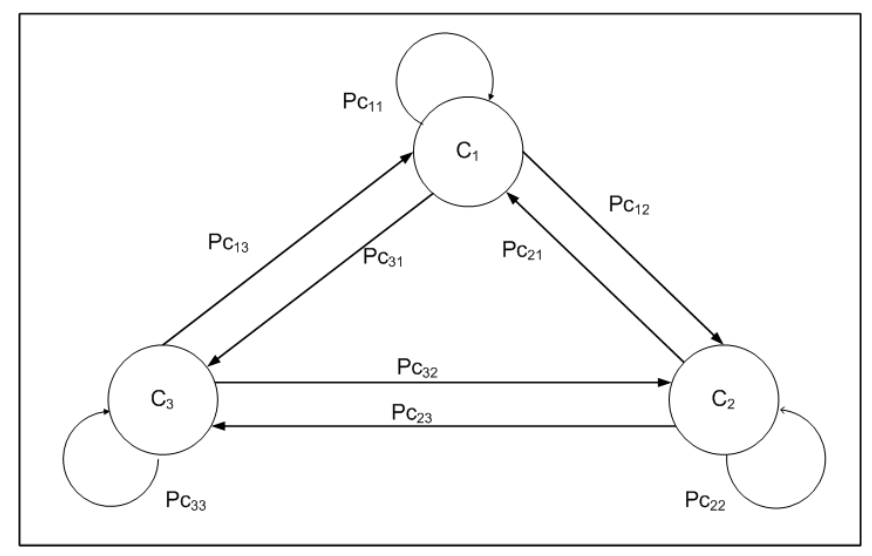

Figure 1: Transition matrix of a node for three communities

\section{A. Deriving the Utility Function Parameters}

To calculate the probability of contact (the utility function) $P_{A B}$, the proposed algorithm PF needs to compute the following parameters: (i) the transition probability matrix, $P^{n}$, (ii) the sojourn time probability distribution matrix, $S_{i}^{A}(k)$, (iii) the fraction of time in which node $A$ resides in community $C_{i}, \pi_{A_{i}}$, which represents the probability distribution at the steady state, (vi) the probability of making successful data exchange between nodes $P_{f}$, (v) the community inter-arrival rate matrix, $\lambda_{i_{A}}$, and (iv) the average residence time of node $A$ in the network, $R_{m}$. These parameters are retrieved from the nodal contact statistics history.

1) Transition Probability Matrix : The illustration of the transition probability matrix, $P_{C}^{A}$, of the embedded Markov chain for node A, that visits three communities is shown in Fig. 1. At any of those communities, the node could choose to stay for a while, or move to another community according to its preferred probability.

For example, if the node is at the $C_{1}$, it can either (1) move to the $C_{2}$ with probability $p_{C_{12}}^{A}$, (2) or stay in $C_{1}$ with the probability $p_{C_{11}}^{A}$, (3) or go to $C_{3}$ with probability $p_{C 13}^{A}$. Those mobility probabilities constitute the transition probability matrix $P_{C_{i j}}^{A}$. Note that each node has its own transition probability matrix at each community that reflects its movement history. The transition probability of node A, $P_{C_{i j}}^{A}$ is calculated by observing all the movement frequency of node $\mathrm{A}$ from $C_{i}$ to $C_{j}$, which is calculated as

$$
p_{C_{i j}}^{A}=\frac{n u m_{i j}^{A}}{n u m_{i}^{A}}
$$

where num $_{i}^{A}$ is the number of all transitions from community $C_{i}$ to any community, and $n u m_{i j}^{A}$ is the number of transitions from community $C_{i}$ to specific community $C_{j}$. By keeping track of $n u m_{i}^{A}$ and $n u m_{i j}^{A}$, each node could generate and refine its own $P_{C_{i j}}$ matrix over time.

2) Probability of Sojourn Time : The probability distribution $S_{i}^{A}(k)$, represents the probability that node $A$ will move from community $C_{i}$ to a community $C_{j}$ at or before time $k$. $S_{i}^{A}(k)$ is based on calculating all residual times node $A$ 
has spend at community $C_{i}$ and followed by transition to a community $C_{j}$, which is defined as following:

$$
S_{i}^{A}(k)=P\left(t_{i j}^{A}<k\right)
$$

where $i_{i j}^{A}$ is the sojourn time at $C_{i}$ when $C_{j}$ is the next visited community. The parameter $k$ is employed as a relative time offset. This distribution is mainly based on the mobility history of a node, which becomes more accurate when the network reaches steady state. The probabilities $P\left(t_{i j}^{m}<k\right)$ for any node $A$ can be obtained by measuring all times $t_{i j}^{A}$ whenever node A moves from community $C_{i}$ to community $C_{j}$. For example, assume that node $A$ has eight measurements for $t_{i j}^{A}$, which are $1,2,5,5,4,6,7,3 . P\left(t_{i j}^{m}<5\right)$ is $1 / 2$ by using the following general formula

$$
P\left(t_{i j}^{A}<k\right)=\sum_{n=0}^{k-1} P\left(A_{i j}^{m}=n\right)
$$

This probability is used to estimate the expected contact time to transfer a message from node $A$ to node $D$.

3) Probability of Steady State: The fraction of time node $A$ resides in community $i, \pi_{A_{i}}$, is calculated as

$$
\pi_{A_{i}}=\frac{\sum_{i} t_{i}^{A}}{\sum_{j} t_{j}^{A}}
$$

where $t_{i}^{A}$ is the duration of time that node A spend at each visit to community $C_{i}$, and $t_{j}^{A}$ is the total time node $A$ spends in all communities at each visit.

4) Community Inter-arrival Time: The community mean inter-arrival rate, $\lambda_{i_{A}}$, is obtained by calculating the residual and roaming times taken by node $A$ while moving from one community to another until it returns back to the same community. Then, (2) is applied to calculate $\lambda_{i_{A}}$.

5) Probability of Successful Data Exchange: As discussed earlier, the probability of making successful data exchange during a contact of any node pair, $P_{f}$, is mainly based on the channel state and the available buffer space, which is in turn largely based on the available bandwidth and the traffic load in the network. $P_{f}$ is calculated by measuring the fraction of time at which the channel is busy or free a contact in average. The statistics of the channel availability is described as follows. The message delivery takes place only when the buffer of the encountered node is not full, and fails otherwise. In case of failure, the time in which the buffer is full during a contact is added to the busy time of the channel, i.e., the busy time is composed of the channel busy time and the time in which the buffer of the encountered node is full during the nodal contact. The statistics of the channel and buffer availability is described as follows.

Let two nodes $A$ and $B$ be in the transmission range of each other, and each broadcasts a pilot signal per $k$ time units in order to look for its neighbors within its transmission range. Let $T_{(A, B)}, T_{\text {free }}$, and $T_{\text {busy }}$ represent the total contact time, the amount of time the channel is free and the buffer is not full, and the amount of time the channel is busy or the buffer is full, respectively, at node $A$ or $B$. Thus, the total duration of time in which node $A$ and $B$ can exchange information is calculated as

$$
T_{\text {free }}=T_{(A, B)}-T_{\text {busy }}
$$

Thus, the probability that the link between node $A$ and node $B$ is available is calculated as following.

$$
P_{f}=\frac{T_{\text {free }}}{T_{(A, B)}}
$$

Whenever node $A$ encounters node $B$ at a community, node $A$ calculates and maintains the $P_{f}$ value regarding node $B$ of that community (i.e, different $P_{f}$ values at different communities). To handle the fluctuation of channel state, when a node roam from one community and enter new one, it broadcasts its latest statistics regarding the channel condition for the community it was in. Then the other nodes update their utility function accordingly.

\section{B. Inter-Nodal Information Exchange}

To estimate the contact probability under the proposed PF algorithm, every node or super node needs to keep track of the contact statistics with every other node in the network. Specifically, the contact statistics is defined as a 6-tuple $<$ nodeI $D, P, S, \lambda, \pi, P_{f}, R_{m}>$, where $P$ is the transition probability matrix, $S$ is the sojourn time probability distribution matrix, $\lambda$ is the inter-arrival rate at each community, $\pi$ is the fraction of time in which node $A$ resides in community $i$, and $P_{f}$ is the probability of the channel availability in each community during a contact. All the aforementioned parameters become more valid when the network approaches to the steady state except for the probability of the channel availability, which needs to be frequently updated since it is largely affected by the traffic load. During each contact, the encountered nodes exchange the information regarding the message destination that they don't have in common. With the update, the custodian node decides whether or not the message should be forwarded to the encountered node, which is mainly based on the forwarding mechanism of PF. This exchange of summary vectors is followed by another important update called transitivity update.

Transitivity update: The proposed algorithm has transitivity property [9], based on the observation that if node $A$ frequently encounters node $B$ and $B$ frequently encounters node $D$, then $A$ has a good likelihood to be able to forward messages to $D$ through $B$. Thus, the congestion history of buffer and link availability of node $B$ should be determined accordingly. In order to maximize the contact probability, $P_{(A, D)}$ and to make any message destined to node $D$ goes through node $B$, a proper update using transitivity property should be made.

To deal with this maximization problem (maximizing $\left.P_{(A, D)}\right)$ using transitivity update, we use the inverse of the probability of contact between two nodes. Using this relation in the transitivity update is simpler and can adaptively update values only when $P_{\left(A_{i} D\right)}<P_{(B, D)}$ in order to ensure that node $A$ reaches $D$ through $B$. Otherwise, if $P_{(A, B)}>P_{(B, D)}$, 
the transitivity property is not useful since node $A$ is a better candidate for forwarding messages directly to node $D$ rather than forwarding them through $B$. The inverse of probability of contact between nodes $A$ and $D, P_{r(A, D)}$, is calculated by $\frac{1}{P_{(A, D)}}$, where $P_{(A, D)}$ is the average probability of contact between node $A$ and $D . P_{r(A, B)}$ and $P_{r(B, D)}$, are obtained using the similar way. The calculation of the $P_{(A, B)}$ is given by equation (7). The new updated parameter, $P_{r(A, D)}$, is calculated as follows:

$$
P_{r(A, D)}^{\text {new }}=w P_{r(A, D)}+(1-w)\left(P_{r(A, B)}+P_{r(B, D)}\right)
$$

where $w$ is a weighting factor calculated from

$$
w=\frac{P_{r(A, B)}+P_{r(B, D)}}{P_{r(A, D)}}
$$

Note that $w$ must be less than 1 ; that is $P_{r(A, D)}>P_{r(A, B)}+$ $P_{r(B, D)}$.

The new probability of contact is obtained by applying the following relation

$$
P_{(A, D)}^{n e w}=\frac{1}{P_{r(A, D)}^{n e w}}
$$

$P_{(A, D)}^{\text {new }}$ represents the new value of $P_{r(A, D)}$ that is obtained from the transitivity update. The introduced transitivity-update rule has great impact on protocol performance.

Note that predicting the future mobility of nodes relies on their trajectory history, which is recorded and disseminated throughout the network in an epidemic fashion or through the supper nodes attached to each community.

\section{Forwarding StRategy IN PF}

PF is a multi-copy DTN routing protocol. At each forwarding step, PF selects the next hop with the highest probability of delivery to the destination. If more than one node are in the transmission range of node $A$, node $A$ selects the next hope by comparing a probability metric (utility function) $U(x)$ for all nodes currently in contact with $A$, denoted as $N_{A}$, and for itself, $x \in\{A\} \cup N_{A}$. This metric indicates the delivery performance to the destination if node $A$ selects node $x$ as the next hop and forwards the message to $x$. The current node then selects the next-hop $h$ as the node for which the delivery probability metric is maximized

$$
h=\arg \max _{x \in\{A\} \cup N_{A}} U(x)
$$

If the selected next hop is the current node $(h=A)$, the message will not be forwarded.

\section{A. Forwarding Strategy}

The decision of message forwarding in PF is mainly based on the utility function value of the encountered node regarding the destination, and the number of message copy tokens. If more than one message copy are currently carried, the weighted copy rule is applied; otherwise the forwarding rule is applied.
1) Weighted Copy Rule: The idea of weighted copy rule was first examined in [27] and [11], and has been proved to achieve improved delivery delay. The proposed PF scheme will also incorporate with such a mechanism.

The source of a message initially starts with $L$ copies. In the event that any node $A$ that has $n>1$ message copy tokens and encounters another node $B$ with no copies with $P_{(B, D)}>P_{(A, D)}$, node $A$ hands over some of the message copy tokens to node $B$ and keeps the rest for itself according to the following formula:

$$
N_{B}=\left\lfloor N_{A}\left(\frac{P_{(B, D)}}{\left.P_{(B, D)}+P_{(A, D)}\right)}\right)\right\rfloor
$$

where $N_{A}$ is the number of message tokens that node $A$ has, $P_{(B, D)}$ is the predicted probability of node $B$ encounters node $D$, and $P_{(A, D)}$ is the predicted probability of contact between nodes $A$ and $D$. This formula guarantees that the largest number of message copies is spread to relay nodes that have better information about the destination node. After $L$ message copies have been disseminated and carried by the encountered custodian nodes, each custodian node carrying the message performs message forwarding according to the forwarding rule as described below.

2) The Forwarding Rule :

- If the destination node is one hop away from an encountered node, the custodian node hands over the message to the encountered node and completes the message delivery.

- If the utility value of the encountered node relative to that of the destination node is greater than that of the custodian node by a threshold value, $P_{t h}$, a custodian node hands over the message to the encountered node.

- If super node $H$ has utility value regarding a node $C$, which is higher than that of node $A$ or $B$ regarding to $D$, and $C$ has higher probability of meeting $D$ than $A$, and $B$ and $H$. Then node $A$ gives the message to $H$.

The complete mechanism of the forwarding strategy in $\mathrm{PF}$ is summarized as shown in Algorithm 1.

\section{Vi. Performance Evaluation}

\section{A. Experiment Setup}

To evaluate the performance of the proposed PF scheme, an adapted DTN simulator based on that in [26] was implemented. In the simulation, a community based DTN model with several predefined communities was established. Nodes usually revolve around these communities. That is, the nodes would stay in the neighborhood of a community, or move to the neighborhood of other communities with their own respective probabilities, which is in turn based on their trajectory history. Two nodes can only communicate when they are associated with the same community. In the simulation, 120 nodes move in a $1200 \mathrm{~m} \times 1200 \mathrm{~m}$ square consisting of 6 identical nonadjacent communities with area of $200 \mathrm{~m}$ x $200 \mathrm{~m}$ for each community. Initially, nodes are uniformly distributed among the communities, and each node prefers to 


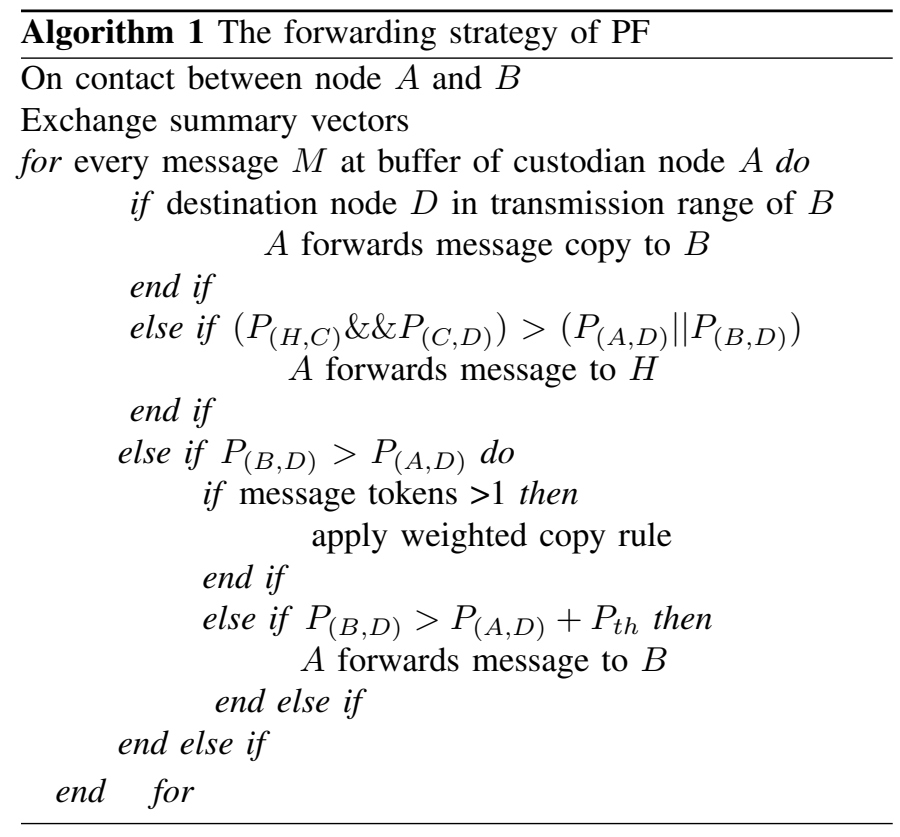

reside at its home community with a longer periods of time than that for any other community.

Each node can transmit up to a distance $K \geq 0$ meters away, and each message transmission takes one time unit. A slotted collision avoidance MAC protocol with Clear-toSend (CTS) and Request-to-Send (RTS), was implemented for contention resolution. A message is acknowledged if it is received successfully at the encountered node by sending back a small acknowledgment packet to the sender.

Each node has its own bounds of sojourn time that it spends at a community, which varies between 5 to 20 time units, with longer periods at its home community. The bounds of roaming time is defined in the range of 100 to 200 time unites, which is the time that a node takes to moves from a community to another. The prediction time window for the PF algorithm is fixed to 300 time units. The choice of the time unit mainly depends on the network scenario and applications under consideration. The PF algorithm has each node to generate the transition probability matrix $P$, steady state probability $\pi$, community inter-arrival rate $\lambda_{i}$, and sojourn time probability distribution $S_{i j}(k)$. Thus, the simulation was run for a "warm-up period" to reach the steady state with stable values of these parameters. The simulation lasted for 30,000 time units in each scenario. The final result is the average of twenty runs.

The message inter-arrival time is uniformly distributed in such a way that the traffic can be varied from low (20 messages per node in 30,000 time units) to high (70 messages per node in 30,000 time units). The message time to live (TTL) is set to 8,000 time units. Each source node selects a random destination node, and begins generating messages to it during simulation time.

The following schemes reported in the literature were also implemented in the simulation for the comparison with the proposed PF scheme.

- Delegation forwarding (DF) [13]

- Spray and focus (S\&F) [15]

- Predict and forward utility-based routing protocol (PF)

- Most mobile first (MMF)[25]

- Self-adaptive routing protocol (SARP) [11]

- PROPHET [9]

For all the protocols, an attempt has been made to tune the parameters in each scenario separately, in order to achieve the best transmission-delay performance. The utility's threshold parameter for Spray and Focus, SARP , and PF was set to 150, 30 , and 0.07 , respectively. Similarly, the number of message copies (L) was set 15 for Spray and Focus, and 10 for SARP and $\mathrm{PF}$.

The performance comparison under the considered mobility scenarios is in terms of average delivery delay, delivery ratio, and the total number of transmissions performed for all delivered messages.

\section{B. Evaluation Scenarios}

We analyze the performance implication of the following. First, the performance of the protocols is evaluated with respect to the low transmission range and varying buffer capacity under high traffic load. Second, with respect to the highlevel of connectivity at each community and varying traffic load. Third, with respect to moderate-level of connectivity and varying message TTL.

1) The Effect of Buffer Size: To examine the effect of buffer sizes upon the performance of $\mathrm{PF}$, the transmission range is set to moderate (i.e., $\mathrm{K}=10$ ) and the traffic load is 60 messages generated per node (relatively high). Fig. 2 shows the experiment results where the buffer space was varied from 5 (very limited capacity) to 200 (relatively high capacity) messages to reflect the performance of the protocols under the considered traffic load. As expected, due to the high traffic volumes, we see a significant impact upon the message forwarding decisions due to the degradation of utility function values caused by buffer overflow. Note that when the buffer of the encountered node is full, some messages cannot be delivered even though the encountered node utility value regarding the message destination is better than that of the custodian node. This situation results in extra queuing delay, especially in the case that flooding-based schemes are in place. As shown in Fig. 2, when the buffer size is small (50 messages or less) the performance of the protocols is very sensitive to the change of buffer capacity.

It is observed that the PF scheme produced the best performance in all scenarios, since it considers the situation that a node may have a full buffer, and then degrades the corresponding utility metric, producing the best performance. In specific, $\mathrm{PF}$ yielded a shorter delivery delay than that of PROPHET by $170 \%$, S\&F by $59 \%$, and SARP by $25 \%$. PF can achieve a higher delivery ratio than DF by $76 \%$, PROPHET by $81 \%$, S\&F by $68 \%$, and SARP by $19 \%$. Although PF produced more transmissions than MMF and DF, it yielded a smaller delivery delay than that of MMF by $84 \%$, and DF by $68 \%$. 


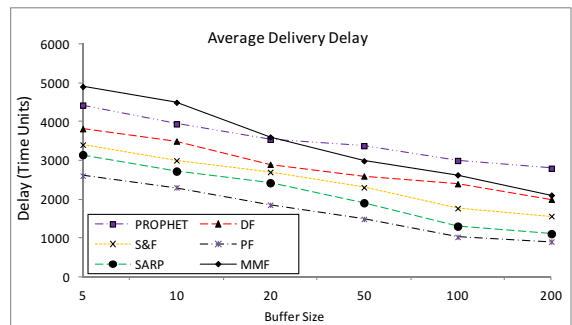

(a)

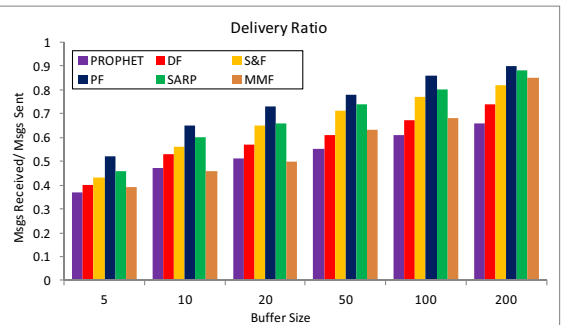

(b)

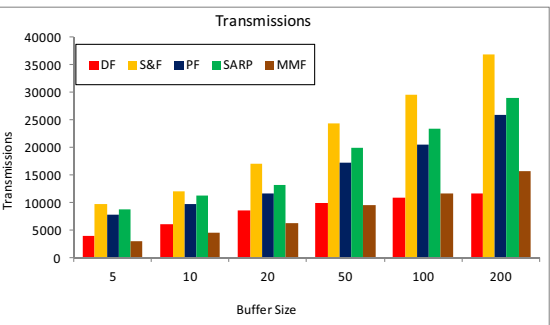

(c)

Figure 2: The effect of buffer size

As the buffer size increased, the performance of all protocols improved especially for MMF and SARP. When the buffer size is larger than the traffic demand, the SARP scheme has yielded a competitive performance due to the relaxation of buffer capacity limitation. PF still yielded the best performance with a smaller number of transmissions than $\mathrm{S} \& \mathrm{~F}$ by $35 \%$.

2) The Effect of Traffic Load : The main goal of this scenario is to observe the performance impact and how PF reacts under different degrees of wireless channel contention. The network connectivity is kept high (i.e., the transmission range is set to as high as 40 meters) under different traffic loads, while channel bandwidth is set relatively quite small (i.e., one message transfer per unit of time) in order to create congested environment. We have two scenarios for nodal buffer capacity: 1) unlimited capacity; and 2) low capacity (15 messages). Fig. 3 shows the performance of all the routing algorithms in terms of the average delivery delay, delivery ratio, and total number of transmissions.

It is observed that PROPHET produced the longest delivery delay and requires a higher number of transmissions compared to all the other schemes, thus it is not included in Fig. 3(c). PROPHET produced an order of magnitude more transmissions than that by PF.

As shown in Fig. 3, when the traffic load is increased, the available bandwidth is decreased accordingly, which causes performance reduction. When the traffic load is moderate (i.e., less that 40 messages), it is clear that the delivery delay is short in all the schemes, while PF outperforms all other protocols and MMF is the second best. This is because in MMF, the effect of buffer size is relaxed, which makes nodes buffer an unlimited number of messages while roaming among communities. PF can produce delays shorter than that of PROPHET, MMF, DF, S\&F, and SARP by $360 \%, 54 \%, 430 \%$, $270 \%$, and $59 \%$, respectively. Regarding the delivery ratio, PF, MMF, S\&F, and SARP can achieve excellent performance of $98 \%$, while the PROPHET routing degrades below $70 \%$ for high traffic loads. DF can achieve delivery ratio above $90 \%$.

As expected, the performance of all the schemes degrades as wireless channel contention is getting higher, especially when the traffic load exceeds 50 messages per node during the simulation period. We observed that PF can achieve significantly better performance compared to all the other schemes, due to the consideration of busy links in its message for- warding mechanism, where the corresponding routing-metric is reduced accordingly. This results in the ability of rerouting the contended messages through the areas of low congestion. However, such a rerouting mechanism makes messages take possibly long routes and results in more transmissions than that of MMF and DF. In summary, the delivery delay obtained by the $\mathrm{PF}$ in this scenario is shorter than that of PROPHET by $330 \%$, MMF by $66 \%$, S\&F by $88 \%$, DF by $233 \%$, and SARP by $30 \%$ respectively. Regarding delivery ratio, PF can achieve as high as $93 \%$, compared with $90 \%$ by SARP, $87 \%$ by MMF, $77 \%$ by $\mathrm{DF}$, and $88 \%$ by S\&F. Even though DF produced the lowest number of transmissions, it is at the expense of the worst delivery delay.

As the buffer capacity is low (e.g., 15 messages) and the traffic load is high, the available bandwidth decreases and the buffer occupancy increases accordingly, which degrades the performance of all protocols, especially for the PROPHET and MMF. It is observed that PROPHET produced the largest delivery delay. It is notable that PF outperforms all the multiple-copy routing protocols in terms of delivery delay and delivery ratio under all possible traffic loads. When the traffic load is high, PF yielded shorter delivery delay than that of SARP by $28 \%$, MMF by $53 \%$, SF by $41 \%$, DF by $47 \%$, and PROPHET by $233 \%$. Although PF requires more transmissions compared to the $\mathrm{MMF}$ and $\mathrm{DF}$, the number is still smaller than that produced by $\mathrm{S} \& \mathrm{~F}$. PF can achieve delivery ratio above $76 \%$ for high traffic loads, while the SARP, PROPHET , DF, S\&F, and MMF degrades by $66 \%$, $47 \%, 51 \%, 62 \%$, and 55\%, respectively. Even though SARP obtains the second best performance, its mechanism is not capable in dealing with the uncertainty of the buffer occupancy status and channel condition, resulting in degradation in its performance. Fig. 4 shows the performance of all techniques under this scenario.

3) The Effect of Message TTL: In this scenario, we study the effect of message TTL, by evaluating the performance of PF under different expiration times. The traffic load is set to 50 message per node, and the buffer size to 15 messages. The main conclusion that can be drawn from Fig. 5 is that; smaller value of TTL leads to lower delivery ratio. This degradation of message delivery is due to the fact that the TTL of a massage might be expired before the message gets propagated to other nodes in order to reach its destination. 


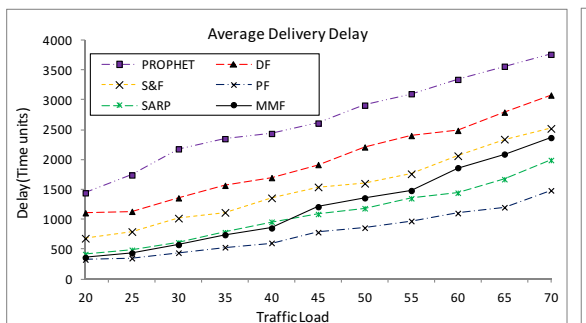

(a)

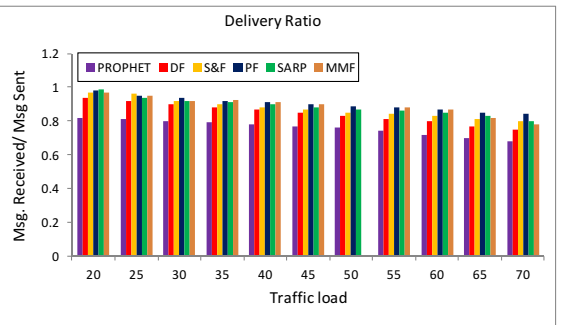

(b)

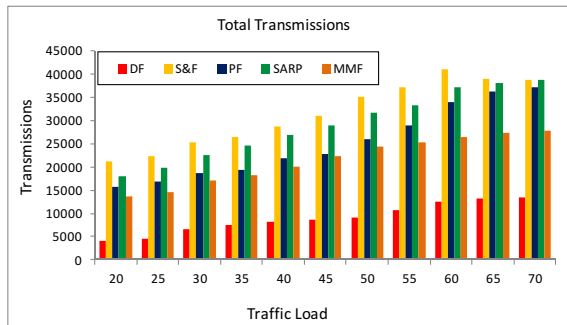

(c)

Figure 3: The effect of traffic load under high buffer capacity

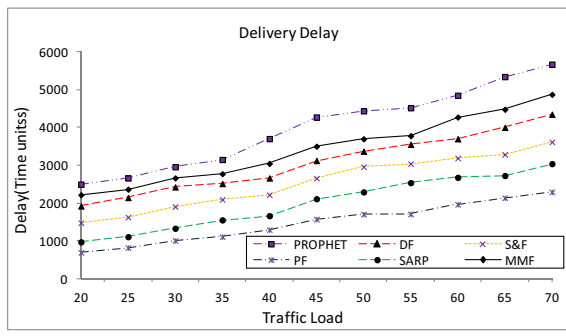

(a)

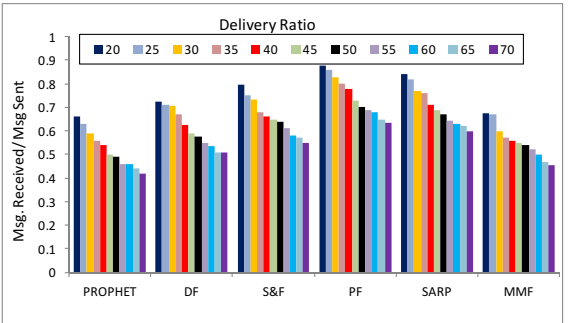

(b)

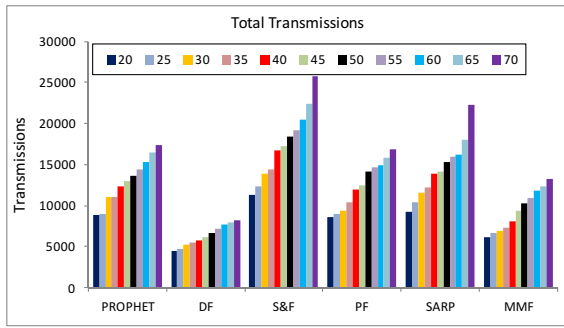

(c)

Figure 4: The effect of traffic load under low buffer capacity

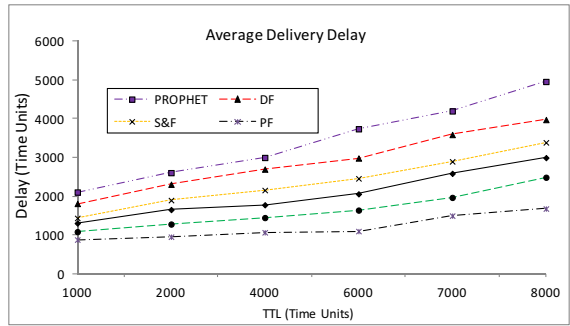

(a)

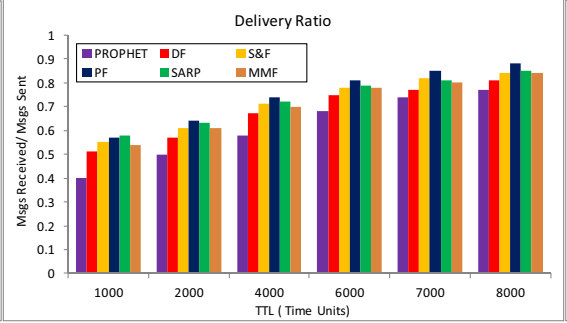

(b)

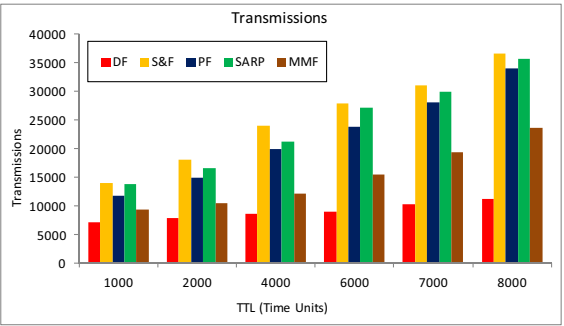

(c)

Figure 5: The effect of message TTL

As the TTL increases, the delivery latency of all protocols increases because more long-lived packets are successfully delivered. Noticeably, PR scheme dominates with the smallest delivery latency. The total number of transmissions produced by each protocol increases as the TTL of messages increases, this due to the increased chance of forwarding messages to more nodes before they get expired or delivered.

\section{CONCLUSION}

The paper introduced a novel multi-copy routing scheme, called PF, for intermittently connected mobile networks that are possibly formed by densely distributed and hand-held devices such as smart phones and personal digital assistants. PF aims to explore the possibility of taking mobile nodes as message carriers in order for end-to-end delivery of the messages. The best carrier for a message is determined by the prediction result using a novel contact model based on the probability distribution of future inter-contact and contact durations, where the network status, including wireless link condition and nodal buffer availability, are jointly considered. We compared PF with a number of counterparts via extensive simulations. It was shown that PF can achieve shorter delivery delays than all the existing spraying and flooding based schemes when the network experiences considerable contention on wireless links and/or buffer space. The study provides a significance that when nodal contact does not solely serve as the major performance factor, the DTN routing performance can be significantly improved by further considering other resource limitations in the utility function and message weighting/forwarding process.

\section{REFERENCES}

[1] Disruption Tolerant Networking. http://www.dtnrg.org.

[2] J. Broch, D. A. Maltz, D. B. Johnson, Y.-C. Hu, and J. Jetcheva, "A performance comparison of multi-hop wireless ad hoc network routing protocols," Proc. ACM MobiCom. Oct. 1998

[3] S. Merugu, M. H. Ammar and E. W. Zegura, "Routing in Space and Time in Networks with Predictable Mobility," Georgia Institute of Technology, Technical Report, GIT-CC-04-7, March 2004. 
[4] S. Jain, K. Fall and R. Patra, "Routing in a Delay Tolerant Network," Proc. ACM SIGCOMM, 2004.

[5] J. Jackson, "The Interplanetary Internet," IEEE Spectrum, Volume 42, Issue No. 8, p. 30, August 2005.

[6] A. Vahdat and D. Becker, "Epidemic Routing for Partially Connected Ad Hoc Networks," Technical Report CS-200006, Duke Univ., Apr. 2000.

[7] Y. Tseng, S. Ni, Y. Chen, and J. Sheu, "The Broadcast Storm Problem in a Mobile Ad Hoc Network," Proc. ACM/IEEE MobiCom, 1999.

[8] T. Spyropoulos, K. Psounis, and C. Raghavendra, "Spray and Wait: An Efficient Routing Scheme for Intermittently Connected Mobile Networks," Proc. ACM SIGCOMM Workshop Delay-Tolerant Networking (WDTN '05), 2005.

[9] A. Lindgren, A. Doria and O. Schelén, "Probabilistic Routing in Intermittently Connected Networks," SIGMOBILE Mobile Computing Comm. Rev., vol. 7, no. 3, pp. 19-20, July 2003.

[10] A. Balasubramanian, B.N. Levine, and A. Venkataramani, "DTN Routing as a Resource Allocation Problem," Proc. ACM SIGCOMM, 2007.

[11] A. Elwhishi and P. Ho, "SARP - a novel multi-copy routing protocol for intermittently connected mobile networks," Proc. IEEE GLOBECOM, 2009.

[12] Q. Yuan, I. Cardei, and J. Wu, "An Efficient Prediction-based Routing Protocol in Disruption-Tolerant Networks", IEEE Trans. Parallel Distrib. Syst. 23, 1 (January 2012), 19-31.

[13] V. Erramilli, M. Crovella, A. Chaintreau , and C. Diot, “ Delegation forwarding," Proc. ACM MobiHoc, 2008.

[14] T. Spyropoulos, T. Turletti, and K. Obraczka, "Routing in delay-toleran networks comprising heterogeneous node populations," IEEE Trans. Mobile Computing, vol. 8, no. 8, pp. 1132-1147, August 2009.

[15] T. Spyropoulos, K. Psounis, and C. S. Raghavendra, "Efficient Routing in Intermittently Connected Mobile Networks: The Single-Copy Case," IEEE Trans. Networking, vol. 16, no. 1, pp. 63-76, Feb. 2008.

[16] S. Nelson, M. Bakht, Robin Kravets, and Albert F. Harris, "Encounter: based routing in DTNs," Proc. IEEE INFOCOM, 2009.

[17] E. Jones, Lily Li, and P. Ward, " Practical Routing in Delay-Tolerant Networks," Proc. ACM SIGCOMM'05 Workshops, 2005.

[18] S. Ling and W. Wei, "Feedback Adaptive Routing Algorithm for DTN," WRI Int'l Conf. on Communications and Mobile Computing, CMC, vol. 2, pp.267-271, 2009.

[19] V. Erramilli, M. Crovella, "Forwarding in opportunistic Networks with Resource constraints," Proc. ACM CHANTS 2008.

[20] Z. Li and Haiying Shen, "Utility-based Distributed Routing in Intermittently Connected Networks," Proc. ICPP, 2008.

[21] M. Musolesi and C. Mascolo, "CAR: Context-aware Adaptive Routing for Delay Tolerant Mobile Networks," IEEE Trans. Mobile Computing. vol.8, no. 2, pp.246-260, Feb. 2009.

[22] A. Elwhishi, Pin-Han Ho, K. Naik, and B. Shihadda, "ARBR: Adaptive Reinforcement-Based Routing for DTN," Proc. IEEE WiMob, 2010.

[23] H. Samuel, W. Zhuang, and B. Preiss, "DTN based dominating set routing for MANET in heterogeneous wireless networking," ACM Mobile Networks and Applications (MONET), vol. 14, no. 2, pp. 154-164, April 2009.

[24] A. Chaintreau, P. Hui, J. Crowcroft, C. Diot, R. Gass, and J. Scott, "Impact of human mobility on opportunistic forwarding algorithms," IEEE Trans. Mobile Computing, vol. 6, no. 6, pp. 606-620, Jun. 2007.

[25] T. Spyropoulos, T. Turletti, and K. Obraczka, "Routing in delaytolerant networks comprising heterogeneous node populations," IEEE Transactions on Mobile Computing, vol. 8, no. 8, pp. 1132-1147, August 2009.

[26] “DTN java simulator" http://people.ee.ethz.ch/ spyropot/dtnsim.html.

[27] Yong Liao, Zhensheng Zhang, Bo Ryu, and Lixin Gao, "Cooperative Robust Forwarding Scheme in DTNs Using Erasure Coding," Proc. IEEE MILCOM, 2007. 